\title{
Phenomenological model for the gravitational-wave signal from precessing binary black holes with two-spin effects
}

\author{
Sebastian Khan, ${ }^{1,2}$ Katerina Chatziioannou, ${ }^{3}$ Mark Hannam, ${ }^{4}$ and Frank Ohme ${ }^{1,2}$ \\ ${ }^{1}$ Max Planck Institute for Gravitational Physics (Albert Einstein Institute), \\ Callinstr. 38, 30167 Hannover, Germany \\ ${ }^{2}$ Leibniz Universität Hannover, D-30167 Hannover, Germany \\ ${ }^{3}$ Canadian Institute for Theoretical Astrophysics, 60 St. George Street, University of Toronto, \\ Toronto, Ontario M5S 3H8, Canada \\ ${ }^{4}$ School of Physics and Astronomy, Cardiff University, The Parade, Cardiff CF24 3AA, United Kingdom
}

(Received 8 October 2018; published 29 July 2019)

\begin{abstract}
The properties of compact binaries, such as masses and spins, are imprinted in the gravitational waves (GWs) they emit and can be measured using parametrized waveform models. Accurately and efficiently describing the complicated precessional dynamics of the various angular momenta of the system in these waveform models is the object of active investigation. One of the key models extensively used in the analysis of LIGO and Virgo data is the single-precessing-spin waveform model IMRPhenomPv2. In this article we present a new model IMRPhenomPv3, which includes the effects of two independent spins in the precession dynamics. Whereas IMRPhenomPv2 utilizes a single-spin frequency-dependent postNewtonian rotation to describe precession effects, the improved model, IMRPhenomPv3, employs a double-spin rotation that is based on recent developments in the description of precessional dynamics. Besides double-spin precession, the improved model benefits from a more accurate description of precessional effects. We validate our new model against a large set of precessing numerical-relativity simulations. We find that IMRPhenomPv3 has better agreement with the inspiral portion of precessing binary-black-hole simulations and is more robust across a larger region of the parameter space than IMRPhenomPv2. As a first application we analyze the gravitational-wave event GW151226 with an efficient frequency-domain waveform model that describes two-spin precession. Within statistical uncertainty our results are consistent with published results. IMRPhenomPv3 will allow studies of the measurability of individual spins of binary black holes using GWs and can be used as a foundation upon which to build further improvements, such as modeling precession through merger, extending to higher multipoles, and including tidal effects.
\end{abstract}

DOI: 10.1103/PhysRevD.100.024059

\section{INTRODUCTION}

Binary systems of compact bodies such as neutron stars (NSs) and black holes (BHs) generate gravitational waves (GWs) that are detectable by second-generation groundbased interferometers. The energy carried away by GWs causes the orbit to decay and the binary to merge on astrophysical time scales. These tiny ripples in spacetime propagate almost completely unaffected through the Universe and have the source properties imprinted in the gravitational waveform. In August 2017 the second observing run (O2) of the detectors aLIGO [1] and Virgo [2] ended, and to date $\mathrm{O} 1$ and $\mathrm{O} 2$ have resulted in the publication of six likely binary black hole $(\mathrm{BBH})$ [3-7] merger events, and the joint GW-electromagnetic observation of a pair of merging NSs $[8,9]$.

Detection and characterization of $\mathrm{GW}$ signals is carried out by a suite of software pipelines that analyze detector data using a variety of analysis methods [10-14].
Matched-filter-based analyses, used in the search for and parameter estimation [15-17] of GW signals from compact binaries, require accurate and computationally inexpensive models for the GW signals, which are used as templates. The need for computationally tractable analyses is best satisfied by frequency-domain models. Moreover, the accuracy of GW signal models and the quantity and quality of the physical effects they include impact both the types of sources GW analyses are sensitive to and the fidelity of the conclusions we draw about their properties.

One such physical effect that has been at the center of waveform-modeling efforts in recent years is spin precession: when the binary components' spin angular momenta are misaligned with the orbital angular momentum of the binary, spin-orbit and spin-spin interactions cause the binary orbit to change orientation in space, resulting in modulations in the observed signal amplitude and phase $[18,19]$. Modeling these modulations is a challenging task, 
especially for systems with unequal-mass components that are observed from close to the binary plane, since this case requires accurate modeling of both spin-precession effects and higher modes beyond the dominant $(\ell,|m|)=(2,2)$ multipoles.

Numerous models have been developed in recent years with the goal of providing a complete coverage across the $\mathrm{BBH}$ parameter space containing all relevant physical effects required for the current sensitivity of GW detectors.

The effective-one-body-numerical-relativity (EOB-NR) model, SEOBNRv3 [20], built upon the nonprecessing model of [21], is a time-domain two-spin precessing model. It provides the $(\ell,|m|) \in((2,2),(2,1))$ multipoles in the coprecessing frame although only the $(2,2)$ is calibrated to numerical-relativity (NR) data. This model has been shown to accurately model the $\ell=2$ multipoles from precessing BBHs [22]; however, as it requires the integration of a set of coupled ordinary differential equations it incurs a large computational cost.

The phenomenological (phenom) waveform models are typically developed and constructed in the frequency domain and the precessing model IMRPhenomP was presented in [23]. IMRPhenomP was built upon the nonprecessing model of [24] and was later upgraded to IMRPhenomPv2, which used the nonprecessing model of $[25,26]$. Despite its computational efficiency IMRPhenomP maps the two spins to one effective spin that governs the precession dynamics, though this choice was shown to not lead to appreciable biases in the characterization of the first $\mathrm{BBH}$ signal [27,28]. A timedomain phenom model for precessing BBHs was presented in [29]; however, it is restricted to equal-mass BBHs with spin magnitudes up to 0.6 .

Recently work has been done to develop models for multipoles beyond the dominant $(\ell,|m|)=(2,2)$ multipole. The first of such models were the nonspinning EOB-NR model of [30] and a nonspinning phenom model was presented in [31]. The first extension of these higher multipole modes into spinning nonprecessing $\mathrm{BBHs}$ has been accomplished both by the phenom [32] and effectiveone-body (EOB) [33] approaches.

An alternative approach to waveform modeling has been successful in building surrogate models [34] that interpolate GW data from NR simulations. The surrogate model of [35] is a fully precessing, time-domain model containing $\ell \leq 4$ multipoles; however, it is restricted to systems where the ratio of the components' masses is less than 2. A hybrid approach to improve existing analytical models by using methods similar to the surrogate modelling has also recently been suggested [36].

In this paper we take another step towards a computationally tractable $\mathrm{GW}$ model that accurately models generic $\mathrm{BBH}$ systems by introducing the frequency-domain fully precessing phenomenological model, IMRPhenomPv3, that describes the inspiral, merger, and ringdown phases of spinning BBHs. Our upgrade from IMRPhenomPv2 to IMRPhenomPv3 hinges on a novel closed-form solution to the differential equations that describe precession including the effects of radiation reaction in the postNewtonian (PN) regime where the binary components are well separated $[37,38]$. This closed-form solution to the precession equations including the effects of radiation reaction has been shown to accurately describe precession for systems with generic masses and spins [37]. Moreover, since it is an analytic frequency-domain model, it is also amenable to the reduced-order-quadrature method to greatly accelerate likelihood evaluations for fast parameter estimation [39]. In addition, IMRPhenomPv2 exhibits nonphysical precession behavior for some high mass-ratio, antialigned spin configurations, as discussed in Sec. II D, but this behavior is not observed in IMRPhenomPv3.

We validate our new model by comparing against a large set of precessing NR waveforms. We find that the improved treatment of the inspiral improves the accuracy of the model for measurement of low-mass systems in LIGOVirgo data. Finally, as a first application and demonstration of our model's readiness we perform a Bayesian parameter estimation analysis (using LALInference [16]) on the GW event GW151226 [4]. This is a $\sim 22 M_{\odot} \mathrm{BBH}$ signal where at least one of the $\mathrm{BHs}$ is measured to have a dimensionless spin magnitude of $\gtrsim 0.2$ at the $99 \%$ credible level. The presence of spin and the low total mass, which implies a large number of GW cycles $(\sim 55)$ measurable by the detector, makes this an ideal candidate to look for evidence of precession. This is the first analysis of GW151226 with an efficient frequency-domain inspiralmerger-ringdown (IMR) precession model with the full four degrees of freedom coming from precession.

\section{BUILDING THE NEW MODEL}

In this section we describe the construction of IMRPhenomPv3 and highlight the improvements compared to IMRPhenomPv2.

\section{A. Precessing BBH phenomenology}

A quasicircular BBH system can be parametrized by only seven intrinsic parameters; the mass-ratio $q=m_{1} / m_{2}{ }^{1}$ and six spin angular momenta $\vec{S}_{1}$ and $\vec{S}_{2}$. In addition, the total mass $M=m_{1}+m_{2}$ can be factored out and systems with different total masses can be obtained with appropriate scaling of $M$. For each BBH we can define a Newtonian orbital angular momentum $\vec{L}$, which is perpendicular to the instantaneous orbital plane and a total angular momentum $\vec{J}=\vec{L}+\vec{S}_{1}+\vec{S}_{2}$.

We classify $\mathrm{BBH}$ systems into different categories according to their spin. Nonprecessing systems have BH

\footnotetext{
${ }^{1}$ We use the convention $m_{1} \geq m_{2}$.
} 
spins that are either aligned or antialigned with $\vec{L}$. Systems with spins (anti-) aligned with $\vec{L}$ plunge and merge at (larger) smaller separations compared to nonspinning binaries, shifting the merger and ringdown part of the signal to (lower) higher GW frequencies. Precessing BBHs are systems with arbitrary $\mathrm{BH}$ spin orientation. Interactions between the $\mathrm{BH}$ spins and $\vec{L}$ introduce a torque on the orbital angular momentum, causing it to precess around the (almost constant) direction of the total angular momentum. Special subcategories of precessing systems include simple- and transitional-precession binaries [18,19]. Most binaries undergo simple precession, while transitional precession occurs when $\vec{J}=\vec{L}+\vec{S}_{1}+\vec{S}_{2} \approx 0$ [18].

\section{B. Modeling precessing BBHs}

The complicated phenomenology of generically precessing BBHs makes waveform modeling especially challenging. A significant advance was achieved when it was observed that the GW signal from precessing binaries is simplified when observed in a frame that is adapted to the precessional motion of the binary [40,41]. In this noninertial (coprecessing) frame the $z$ axis approximately tracks the orientation of the orbital plane. When the waveform is transformed into this frame it closely mimics the signal of the equivalent $\mathrm{BBH}$ system that has the spin components perpendicular to $\vec{L}$ set to $0[40,42]$. This is a consequence of the approximate decoupling between the components of spin parallel and perpendicular to $\vec{L}$; the former influences the rate of inspiral and the latter drives the precessional motion $[18,40,43]$.

This observation led to a method for building models for the gravitational waveform from generic precessing BBHs by first constructing a model for the gravitational waveform produced by the equivalent nonprecessing $\mathrm{BBHs}$ and then introducing precession through a time- (or frequency-) dependent rotation of the signal derived from the orbital dynamics [44]. Colloquially this procedure is denoted "twisting-up" a nonprecessing model to produce a precessing model [44].

Beginning with an inertial frame that is aligned with the total angular momentum at some reference frequency $\hat{z}=\hat{J}$ we describe the orbital angular momentum by the azimuthal and polar angles $(\alpha, \beta)$. In order to completely specify the rotation we use the frame that minimizes precessional effects and adopt the "minimum rotation condition" [45], which enforces the third Euler angle to obey $\dot{\epsilon}(t)=$ $\dot{\alpha}(t) \cos (\beta(t))$. This angle constitutes a modification to the orbital phase chosen so that the frequency in the inertial frame is the same as the frequency in the coprecessing frame. For a geometric depiction, see Fig. 1 in Ref. [37]. ${ }^{2}$

\footnotetext{
${ }^{2}$ To convert from the notation used in [37] to ours, use the following substitutions: $\phi_{z} \rightarrow \alpha, \theta_{L} \rightarrow \beta$ and $\zeta \rightarrow \epsilon$.
}

This procedure was used to produce the first precessing IMR models [20,21,23]. The general procedure is as follows. First, we express the two GW polarizations $h_{+}$ and $h_{\times}$as a linear combination of spherical harmonics with spin weight -2 . The coefficients of the basis functions are the GW multipoles $h_{\ell, m}$; see Eq. (1). This decomposition is performed in a frame that is aligned with the total angular momentum and the direction of propagation is given by the spherical polar coordinates $(\theta, \varphi)$,

$$
h(t ; \theta, \phi)=h_{+}-i h_{\times}=\sum_{\ell \geq 2, m} h_{\ell, m}(t) Y_{\ell, m}^{-2}(\theta, \varphi) .
$$

From here we wish to express the GW multipoles from precessing BBHs $h_{\ell, m}^{\text {prec }}$ in terms of the multipoles from nonprecessing BBHs $h_{\ell, m}^{\text {ron }}$ and the appropriate angles that describe the twisting-up from nonprecessional to precessional dynamics. This is done by applying the Wigner-D rotation matrices to the GW multipoles from the nonprecessing system using the angles $(\alpha, \beta, \epsilon)[40,41,44,45]$. Here we focus on the $\ell=2$ multipoles and the case where the nonprecessing model only contains the $\ell=|m|=2$ multipoles. In that case, the waveform is given by

$$
h_{2, m}^{\mathrm{prec}}(t)=e^{-i m \alpha(t)} \sum_{\left|m^{\prime}\right|=2} e^{i m^{\prime} \epsilon(t)} d_{m^{\prime}, m}^{2}(-\beta(t)) h_{2, m^{\prime}}^{\substack{\text { non } \\ \text { prec }}}(t) .
$$

In order to complete the transformation from a nonprecessing IMR model to a precessing IMR model we also need to modify the mapping between the inspiraling progenitor $\mathrm{BHs}$ and the final $\mathrm{BH}$, taking into account the effect of precession. We typically assume that changes in the GW flux due to precession can be neglected so that the estimate of the final mass can be taken from fits to NR simulations of nonprecessing systems. The final spin, however, is sensitive to precession and therefore needs to be modified from the model used in the underlying nonprecessing model.

In the next sections, we review the angles $\left(\alpha_{\mathrm{v} 2}, \beta_{\mathrm{v} 2}, \epsilon_{\mathrm{v} 2}\right)$ that were used for the IMRPhenomPv2 model and then we describe the updated $\left(\alpha_{\mathrm{v} 3}, \beta_{\mathrm{v} 3}, \epsilon_{\mathrm{v} 3}\right)$ that we employ to produce the updated model IMRPhenomPv3.

\section{Review of IMRPhenomPv2}

The method described in the previous section to construct a complete IMR model for precessing BBHs was used to create the IMRPhenomP model [23]. The original model used the IMRPhenomC [24] model to describe the nonprecessing system, but was subsequently enhanced to IMRPhenomPv2, which uses IMRPhenomD $[25,26]$, a more accurate aligned-spin model valid for larger mass-ratio binaries and BHs with larger spin magnitudes. Moreover IMRPhenomD includes some two-spin information during the inspiral. Both of these underlying 
aligned-spin models provide only the $\ell=|m|=2$ spherical harmonic multipole.

In both previous versions of IMRPhenomP the precession angles $\left(\alpha_{\mathrm{v} 2}, \beta_{\mathrm{v} 2}, \epsilon_{\mathrm{v} 2}\right)$ were computed by a closed-form frequency-domain expression derived under the assumption of a single-spin system and parametrized by the aligned effective-spin parameter $\chi_{\text {eff }}$ and the precession effective-spin parameter $\chi_{p}$ [46] defined as

$$
\chi_{p}:=\frac{\max \left(A_{1} S_{1 \perp}, A_{2} S_{2 \perp}\right)}{A_{1} m_{1}^{2}},
$$

where $A_{1}=2+\frac{3 m_{2}}{2 m_{1}}, A_{2}=2+\frac{3 m_{1}}{2 m_{2}}$ and $S_{i \perp}$ are the magnitudes of the spin-angular momenta perpendicular to $\vec{L}$. Additionally, IMRPhenomP used a small-angle approximation for the opening angle $(\beta)$, an assumption that was subsequently relaxed in IMRPhenomPv2.

The expressions for $\left(\alpha_{\mathrm{v} 2}, \beta_{\mathrm{v} 2}, \epsilon_{\mathrm{v} 2}\right)$ were derived under the stationary phase approximation (SPA) [47] and are used to twist-up the entire waveform, including through merger and ringdown, where the assumptions of SPA are formally invalid. Nevertheless the extrapolation of the PN expressions into the merger and ringdown has been shown to not impair the model, so long as the mass ratio and spin are not large; see [23] and Sec. III.

IMRPhenomPv2 uses a single-spin approximation that only models the simple-precession case where the direction of $J$ is relatively constant during the inspiral, and the direction of the final BH spin is assumed to be parallel to $J$. The magnitude of the final spin angular momentum $S_{f}$ is computed as the sum of parallel and perpendicular angular momentum components with respect to $\vec{L}$. $S_{\text {non }}$ is the angular momentum from the nonprecessing system and $S_{\perp}$ is the angular momentum of the in-plane spin components.

$$
S_{f} \equiv M_{f}^{2} \chi_{f}=\sqrt{S_{\perp}^{2}+S_{\substack{\text { nonce } \\ \text { prec }}}^{2}}
$$

The final dimensionless spin magnitude $\chi_{f}$, with a free parameter $\lambda$, is written as $[48,49]$

$$
\chi_{f}=\sqrt{\left(S_{\perp} \frac{\lambda^{2}}{M_{f}^{2}}\right)^{2}+\chi_{\substack{\text { nonc } \\ \text { prec }}}^{2}}
$$

The choice made for $\lambda$ in IMRPhenomPv2 is $\lambda=M_{f} / M$, implying that the $S_{\perp}$ angular momentum gets scaled by the initial total mass. We comment that this is a fairly arbitrary choice as we have a model for $M_{f}$ that we use in the nonprecessing part [25]. However, it was found that this simplification still yielded a model with acceptable accuracy. Finally, $S_{\perp}$ is approximated using the effective precessing parameter $\chi_{p}$ and computing the angular momentum by assuming only the primary $\mathrm{BH}$ has in-plane spin components, i.e.,

$$
S_{\perp}=m_{1}^{2} \chi_{p} .
$$

The model IMRPhenomPv2 has been used in numerous publications to estimate the source parameters of all $\mathrm{BBH}$ observations in the LIGO-Virgo $\mathrm{O} 1$ and $\mathrm{O} 2$ runs, e.g., [4-7,50-53], and has been the basis of a reduced order quadrature approximation to the likelihood [39] that has enabled fast parameter estimation of GW sources [54]. The model's low computational cost has also enabled it to form the basis of the model IMRPhenomPv2_NRTidal [55] used for the analysis of the long binary neutron star signal GW170817 [56,57].

\section{Issues with IMRPhenomPv2 precession angles}

Despite IMRPhenomPv2's good performance across the parameter space, there are some known issues with the precession angles. Specifically, the angle $\alpha_{\mathrm{v} 2}(f)$ is written as a power series in $f$, including terms up to next-to-nextto-leading order (NNLO) in the spin. The leading-order Newtonian term (see, for example, Ref. [18]) is

$$
\alpha_{\mathrm{v} 2}^{N}=\alpha_{0}-\frac{20\left(1+\frac{3}{4 q}\right)}{192 f},
$$

where $\alpha_{0}$ is a constant. At this order, the precession angle is a monotonically increasing function, which is what we expect in a physical system exhibiting simple precession: the orbital angular momentum vector precesses around the total angular momentum throughout the binary's evolution, and the rate of precession, steadily increases. At leading order [18] we also see that the precession angle (and therefore also the precession frequency) depend only on the mass ratio of the binary; the spin affects the precession rate at higher orders.

If we extend this expression up to $f^{1 / 3}$, as is done in the NNLO expressions used in IMRPhenomPv2 [58] [which also include a $\log (f)$ term], then higher order terms can enter with opposing signs. In some cases this can remove the monotonicity of $\alpha_{\mathrm{v} 2}(f)$, even for simple-precession configurations where we know that such behavior is not physically consistent.

Figure 1 shows examples of this behavior. We first consider a binary that is in the range of possible configurations identified in GW observations: the mass ratio is 1 , the component of the spin parallel to the orbital angular momentum is 0 , i.e., $\chi_{\text {eff }}=0$, and the larger $\mathrm{BH}$ has a spin of magnitude 0.9 lying in the orbital plane, i.e., $\chi_{p}=0.9$; since $\chi_{p}$ has not been constrained in observations to date, a value of 0.9 is consistent with the measured parameters. The angle of the spin in the plane at a particular reference frequency determines the constant $\alpha_{0}$, but we focus here on the frequency evolution of the precession angle. We plot the precession angle $\alpha_{\mathrm{v} 2}(f)-\alpha\left(f_{0}\right)$, where $M f_{0}=0.0001$, which corresponds to approximately $2 \mathrm{~Hz}$ for a binary with a total mass of $10 M_{\odot}$. The results are shown up to the Schwarzschild ISCO frequency $(M f=0.0217)$. We can see that $\alpha_{\mathrm{v} 2}(f)$, as predicted by the leading-order 

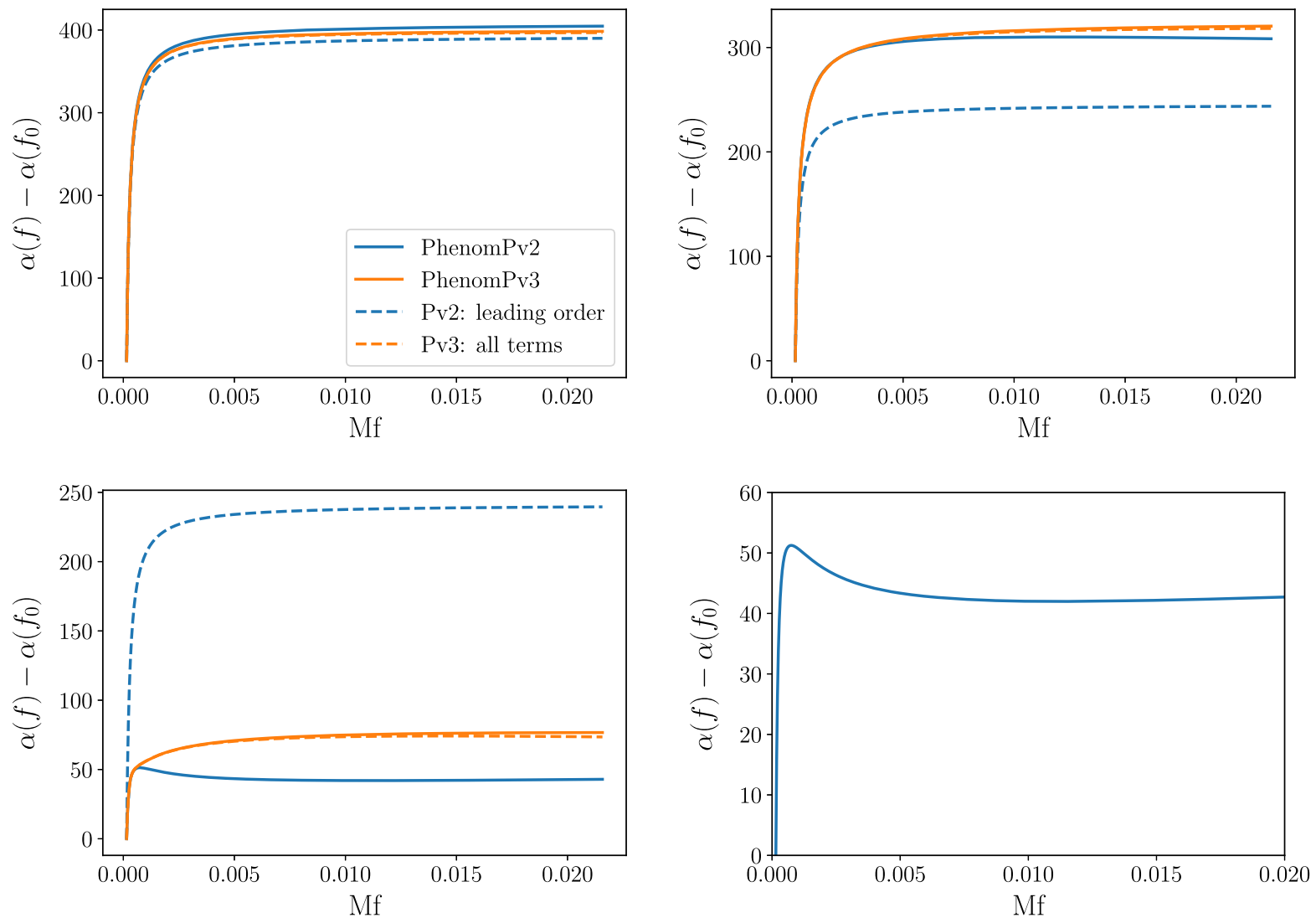

FIG. 1. Comparison of leading-order (IMRPhenomPv2, leading order), NNLO (IMRPhenomPv2), full-PN (IMRPhenomPv3, all terms), and the version we adopt in the final model (IMRPhenomPv3) expressions for the precession angle, $\alpha$. First panel, $q=1, M_{\mathrm{tot}}=10 M_{\odot} \chi_{\mathrm{eff}}=0, \chi_{p}=0.9$; second panel, $q=8, M_{\mathrm{tot}}=10 M_{\odot} \chi_{\mathrm{eff}}=0, \chi_{p}=0.9$; third panel, $q=10, M_{\mathrm{tot}}=$ $10 M_{\odot} \chi_{\text {eff }}=-0.8, \chi_{p}=0.1$; fourth panel, same configuration as third panel, but zoom of nonphysical behavior of $\alpha_{\mathrm{v} 2}(f)$. All cases are generated from a frequency of $2 \mathrm{~Hz}$. See the text for discussion.

Eq. (7) (blue dashed line), the full NNLO expressions used for IMRPhenomPv2 (blue solid line), the full IMRPhenomPv3 expression (orange dashed line), and the truncated IMRPhenomPV3 expression (orange solid line) all agree well, even though spin effects are not included in the leading-order result.

In the second example, we can see that the NNLO expression is needed to accurately describe the precession for higher mass ratios and higher spins. This example shows $\alpha_{\mathrm{v} 2}(f)-\alpha\left(f_{0}\right)$ for a binary with mass ratio 8; again $\chi_{\mathrm{eff}}=0$ and $\chi_{p}=0.9$. The NNLO IMRPhenomPv2 and the IMRPhenomPv3 expressions agree well, but the leadingorder expression accumulates a difference against the others of $\sim 60 \mathrm{rad}$, or $\sim 10$ precession cycles, out of $\sim 47$ cycles over the course of the entire inspiral. This level of agreement between the IMRPhenomPv2 and IMRPhenomPv3 expressions is typical across all configurations with mass ratios up to $\sim 5$, so we can be confident that IMRPhenomPv2 accurately models the precession angle $\alpha_{\mathrm{v} 2}$ with sufficient accuracy for aLIGO and Virgo BBH observations to date.

In the third example, the mass ratio is 10 , but now $\chi_{\text {eff }}=-0.8, \chi_{p}=0.1$. Now the IMRPhenomPv2 expressions disagree significantly between each other and against the IMRPhenomPv3 expressions. Most notable, however, is that the IMRPhenomPv2 precession angle (shown alone in the fourth panel of Fig. 1) reaches a maximum and then decreases; the maximum implies that at this point the precession comes to a halt, and then continues in the opposite direction. This occurs at a frequency of $\sim 10 \mathrm{~Hz}$ for a $10 M_{\odot}$ binary, i.e., many orbits before merger, while the system should still be undergoing simple precession, and when PN results should still be valid. Indeed, simple precession does continue through these frequencies in an evolution of the PN equations of motion, as indicated by the $\alpha_{\mathrm{v} 3}(f)$ results shown in the third panel. We therefore conclude that the NNLO frequency expansion behaves unphysically for certain mass-ratio and spin combinations, and degrades the accuracy of a model in these regions of parameter space.

\section{E. Upgrading to IMRPhenomPv3}

The first closed-form analytic inspiral waveform model for generically precessing BBHs was presented in $[37,38]$. The solution utilized two ingredients. First, the analytic 
solution to the conservative precession equations constructed in [59] was supplemented by radiation-reaction effects through a perturbative expansion in the ratio of the precession to the radiation reaction time scale $[60,61]$ known as multiple scale analysis [62]. Second, the frequency-domain waveform was analytically computed through the method of shifted uniform asymptotics, first introduced in [63]. The resulting inspiral waveform model for the precession dynamics incorporates spin-orbit and spin-spin effects to leading order in the conservative dynamics and up to $3.5 \mathrm{PN}$ order in the dissipative dynamics ignoring spin-spin terms.

We here use the first of the above ingredients, namely, the generic two-spin solution of $[37,38]$ to obtain twospin expressions for the precession angles $\left(\alpha_{\mathrm{v} 3}, \beta_{\mathrm{v} 3}, \epsilon_{\mathrm{v} 3}\right)$. Specifically we use Eqs. (58), (66), and (67) and the coefficients in Appendix D in [37] for $\alpha_{\mathrm{v} 3}$ (denoted as $\phi_{z}$ in that paper). Appendix $\mathrm{F}$ in [37] provides the analogous equations and coefficients for the $\epsilon_{\mathrm{v} 3}$ angle (called $\zeta$ in [37]), while the $\beta_{\mathrm{v} 3}$ angle (called $\theta_{L}$ in [37]) is given by Eq. (8).

As an illustration of the new waveform model we compare IMRPhenomPv2 (blue-dashed) and IMRPhenomPv3 (orange-solid) for a mass ratio 1:10, two-spin system in Fig. 2. The top left panel shows the $h_{\times}(t)^{3} \mathrm{GW}$ polarization viewed at an inclination angle ${ }^{4}$ of $90 \mathrm{deg}$. The top right panel is a zoom in around the merger. At early times both models are in agreement; however, due to the difference between the precession angle models the two models start to noticeably disagree around $90 \mathrm{~s}$ before merger.

Similarly to the angles $\alpha_{\mathrm{v} 2}$ and $\epsilon_{\mathrm{v} 2}$, the expressions for $\alpha_{\mathrm{v} 3}$ and $\epsilon_{\mathrm{v} 3}$ involve series expansions in terms of the GW frequency. The expression for $\epsilon_{\mathrm{v} 3}$ is fully expanded and expressed in terms of a power series of the GW frequency, $f^{-4 / 3+n / 3}, n \in[1,6]$. The angle $\alpha_{\mathrm{v} 3}$ involves both expanded $(n \in[1,6])$ and unexpanded terms, a choice made to increase the angle's accuracy for unequal-mass systems, as discussed in Sec. IV D 1 of [37]. The order to which we truncate the relevant expansions can impact the accuracy of the model: too few $n$ terms and the expansion fails to accurately describe the inspiral but too many $n$ terms and the expressions become inaccurate when extrapolating towards higher frequencies. Figure 2 illustrates the impact of expansion order $n$ on the precession angles. The lowerleft and lower-middle panels show the $\alpha_{\mathrm{v} 3}$ and $\epsilon_{\mathrm{v} 3}$ angles as a function of the $\mathrm{GW}$ frequency. The solid-orange line shows the model that is used in IMRPhenomPv3 and the paler curves show different the result for truncation orders

\footnotetext{
${ }^{3}$ The time domain $h_{\times}(t)$ was obtained by computing the inverse Fourier transform of $\tilde{h}_{\times}(f)$.

${ }^{4}$ Here, inclination is the angle between the initial $\vec{L}$ and the line of sight. Because $\vec{L}$ is evolving on the precession time scale, we emphasize that the value of the inclination angle is more ambiguous than in the nonprecessing case.
}

for the $\alpha_{\mathrm{v} 3}$ and $\epsilon_{\mathrm{v} 3}$ models. The dashed-blue line shows the results for the precession angle model used in IMRPhenomPv2, which deviates away from the other approximations. The $\alpha_{\mathrm{v} 2}$ angle in particular shows qualitatively different behavior, growing rapidly as the frequency increases.

We also have a choice when computing $\beta_{\mathrm{v} 3}$, the angle between $\vec{J}$ and $\vec{L}$. We investigated either using Newtonian order, 2 PN nonspinning (as was done in IMRPhenomPv2) and also a 3 PN version including spin-orbit terms for the magnitude of $\vec{L}$ that is used in the computation of $\beta_{\mathrm{v} 3}$. A comparison between the different methods for calculating $\beta_{\mathrm{v} 3}$ can be seen in the lower-right panel in Fig. 2. The observed modulations are nutation due to spin-spin effects.

The three vertical black lines (from left to right) are the hybrid-MECO [64], Schwarzschild ISCO and the ringdown frequency for this system. We assume the limit to which PN results can be reliably used to be near the hybrid-MECO/ Schwarzschild ISCO and the region between this and the ringdown frequency to be the region where we are extrapolating the precession angles beyond their assumed region of validity. This assumption appears to hold as it seems to track the location of a turnover point in many of the precession angle variants, a feature that is unphysical (at least in simple precession cases) as discussed in Sec. II D.

To decide which choices when computing the precession angles lead to the most accurate model we performed several mismatch calculation comparing different versions of the model with NR. We find that most choices lead to reasonably accurate models for the inspiral, but can lead to suboptimal performance during the merger. We obtain an accurate model for the entire coalescence by using all but the highest order terms in the expressions for $\alpha_{\mathrm{v} 3}$ and $\epsilon_{\mathrm{v} 3}$ and use the highest order PN calculation available, namely, with $3 \mathrm{PN}$ with spin-orbit terms when computing $|\vec{L}|$ in the $\beta$ angle. In Sec. III we only show results for the final model.

The final prescription we need to complete the model is a description of the remnant black hole. We find the method used in IMRPhenomPv2 to be adequate and continue to use it in IMRPhenomPv3. Improvements to this simple model could take the form of implementing explicit models for the remnant black hole; see [65-67].

We also note one other improvement on IMRPhenomPv2, which deals with superkick configurations [68]. These are equal-mass configurations where each black hole has the same spin, but the spins both lie in the orbital plane and point in opposite directions. These configurations possess symmetry such that the orbital plane does not precess, but instead "bobs" up and down as linear momentum is radiated perpendicular to the orbital plane. If IMRPhenomPv2 is given the parameters for such a configuration, the definition of $\chi_{p}$, Eq. (3) will yield a nonzero value, and the code will construct the waveform for a precessing system. Neighboring configurations in parameter space 

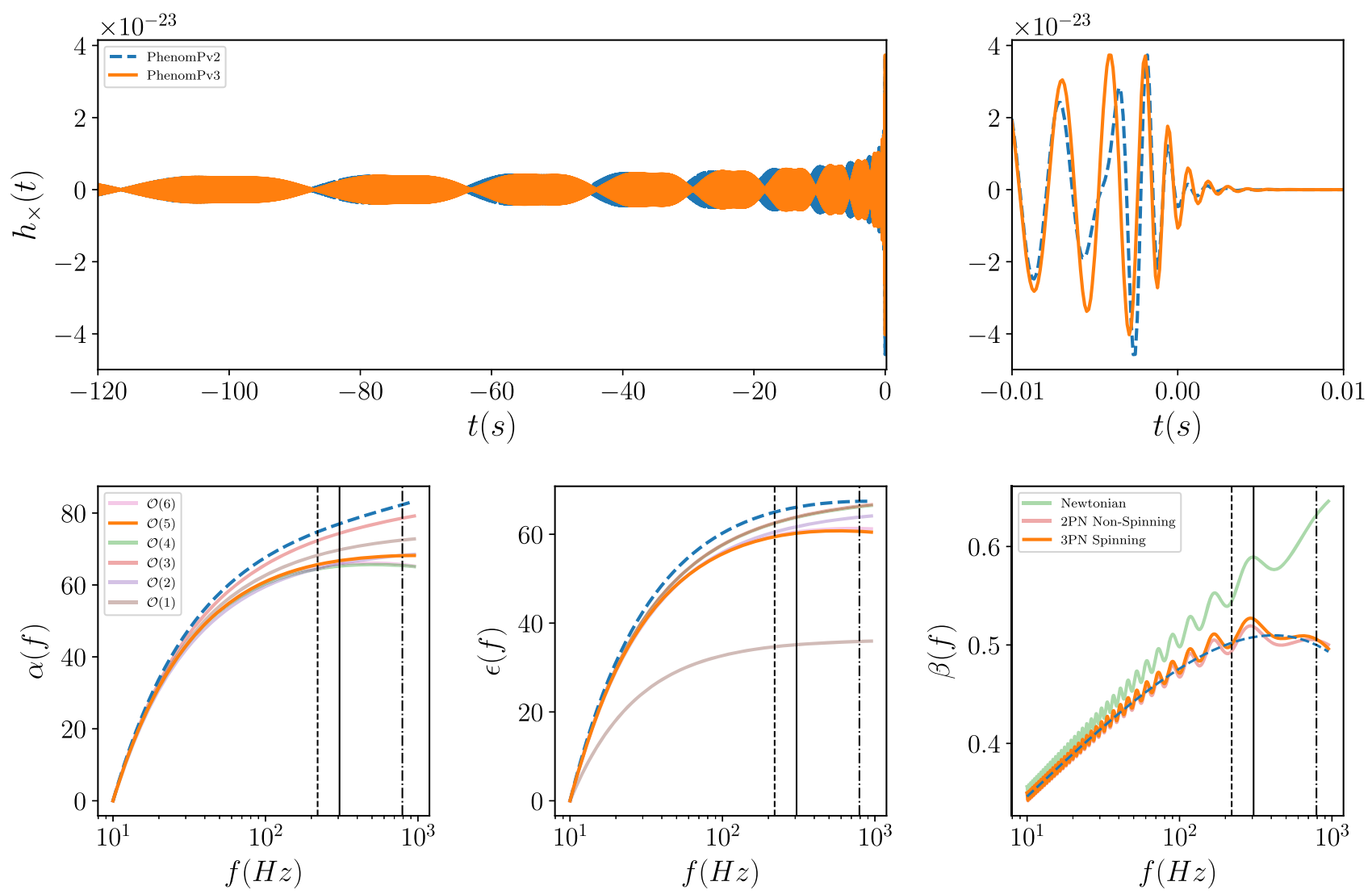

FIG. 2. Composite figure comparing the various models for the precession angles and their overall effect on the waveform. We generate the gravitational waveform from a BBH system with the following parameter: $q=10, M=20, S_{1}=(0.4,0,0.4)$, $S_{2}=(0.3,0,-0.3), f_{\text {start }}=10 \mathrm{~Hz}$. The top row shows $h_{\times}$polarization evaluated at $\iota=\pi / 2$ (here $\imath$ is the angle between $\vec{L}$ and the line of sight). In all panels the dashed blue line corresponds to IMRPhenomPv2 and the solid orange line corresponds to IMRPhenomPv3. Top left: The full waveform generated from $10 \mathrm{~Hz}$. Top right: Zoom in around merger. We observe early-time agreement and late-time disagreement caused by the different precession models. The bottom row shows the precession angles as a function of GW frequency. Bottom left: $\alpha$. Bottom middle: $\epsilon$. Bottom right: $\beta$. The solid black line is the hybrid-MECO frequency [64], the dashed black line is the Schwarzschild ISCO frequency and the dot-dashed black line is the ringdown frequency for this system. The legend in the bottom left plot applies to the bottom middle plot as well and shows to what $n$ order (see text) the $\alpha$ and $\epsilon$ angles were expanded. The legend in the bottom right figure indicates if aligned spins were included in the calculation of the magnitude of the orbital angular momentum.

display similar behavior, and IMRPhenomPv2 again generates the "wrong" waveform. By correctly accounting for both spins, IMRPhenomPv3 corrects this problem. A request for an equal-mass superkick configuration yields the waveform for an equal-mass nonspinning configuration, which is the closest approximation that does not experience recoil.

\section{COMPARISON TO NUMERICAL RELATIVITY}

In this section we compare IMRPhenomPv3 to a large number of NR simulations of precessing $\mathrm{BBHs}$ and show the excellent agreement between our model and the simulations.

\section{A. Mismatch calculation}

In GW searches and parameter estimation, template waveforms are correlated with detector data. This operation can be written as an inner product weighted by the sensitivity of the detector [described by the power spectral density (PSD) $S_{n}(f)$ ] between the real valued template $h(t)$ and signal $s(t)$ waveforms. We define the overlap between the template and signal as

$$
\mathcal{O} \equiv(h \mid s) \equiv 4 \operatorname{Re} \int_{f_{\min }}^{f_{\max }} \frac{\tilde{h}^{*}(f) \tilde{s}(f)}{S_{n}(f)} d f,
$$

where $\tilde{h}$ represents the Fourier transform of $h$ and $\tilde{h}^{*}$ is the complex conjugate of $\tilde{h}$.

This inner product is closely related to the definition of the matched-filter signal-to-noise ratio (SNR) and indeed the $1-\mathcal{O}$ between normalized waveforms $(\hat{x}=x / \sqrt{(x \mid x)})$ is directly proportional to the loss in SNR, making it a useful metric to measure the accuracy of waveform models. 
Aside from the masses and spins of the BHs the GW signal depends on a number of extrinsic parameters: the direction of propagation in the source frame $\left(l, \phi_{0}\right)$, a polarization angle $\psi$, a reference time $t_{0}$ and the luminosity distance $D_{L}$. The dependency of the observed GW signal on the polarization angle (ignoring the angular position of the source with respect to a detector) is given by

$$
h(t)=h_{+}(t) \cos (2 \psi)+h_{\times}(t) \sin (2 \psi) .
$$

Given two waveforms $h$ and $s$ we quantify the agreement between them by computing their normalized overlap subject to various averages and optimizations of extrinsic parameters while keeping the intrinsic parameters fixed. Note that the use of normalized waveforms $\hat{h}$ and $\hat{s}$ factors away the dependency of the $D_{L}$ in the overlap.

In particular for each inclination angle $\imath$ considered we compute the overlap between $\hat{h}$ and $\hat{s}$ with the same masses and spins and vary the extrinsic signal parameters $\left(\phi_{0}^{s}, \psi_{0}^{s}\right)$ by evaluating them on a $10 \times 10$ grid with the following domain: $\phi_{0}^{s} \in[0,2 \pi]$ and $\psi_{0}^{s} \in[0, \pi / 4]$. For each point on this grid we analytically maximize over a time shift using an inverse Fourier transform, analytically maximize over $\psi_{0}^{h}$ according to the method detailed in [69] and finally numerically optimize the overlap over $\phi_{0_{5}}^{h}$ using optimization routines from the SciPy package. ${ }^{5}$ From here we define the match as a function of the extrinsic parameters of the signal as

$$
\mathcal{M}\left(\phi_{0}^{s}, \psi_{0}^{s}\right) \equiv \max _{t_{0}^{h}, \phi_{0}^{h}, \psi_{0}^{h}}\left(\hat{h} \mid \hat{s}\left(\phi_{0}^{s}, \psi_{0}^{s}\right)\right)
$$

Next we average over the extrinsic parameters of the signal $\left(\phi_{0}^{s}, \psi_{0}^{s}\right)$ weighted by its optimal SNR $\rho$ at each point which accounts for the likelihood that this signal would be detected $[43,70]$. We call this the orientationaveraged match,

$$
\overline{\mathcal{M}} \equiv\left(\frac{\sum_{i} \rho_{i}^{3} \mathcal{M}_{i}^{3}}{\sum_{i} \rho_{i}^{3}}\right)^{1 / 3} .
$$

From here we define the orientation-averaged mismatch as $1-\overline{\mathcal{M}}$. We quote results in terms of this. In what follows we use $h$ to denote a template waveform, i.e., generated by a waveform approximant and $s$ to denote the signal waveform which is a NR waveform.

We compute the orientation-averaged mismatch between the template waveform and each NR waveform at three different inclination angles $(0, \pi / 3, \pi / 2)$ rad. By increasing the inclination angle we tend to observe a weaker but more modulated waveform due to the precession.

\footnotetext{
${ }^{5}$ Because we are comparing generic precessing waveforms we cannot analytically optimize over $\phi_{0}^{h}$ in the presence of $m \neq 2$ multipoles.
}

We therefore expect the accuracy of the models to decrease at inclined orientations where the effects of precession are typically more pronounced. We consider three different waveform approximants: IMRPhenomPv2, our improvement IMRPhenomPv3, and the precessing IMR EOB-NR model SEOBNRV3 [22]. For each NR simulation we generate a template with the same masses and spins beginning from the start frequency as reported in the NR metadata. Waveforms were generated using the LALSimulation package, part of the software library LALSuite [71], using the NR injection infrastructure presented in [72].

When comparing GW signal models with NR there is an ambiguity that one encounters when trying to identify a time (or frequency) in the gravitational waveform and the corresponding retarded time (or frequency) of the $\mathrm{BBH}$ dynamics, where spins are measured and defined in the NR simulation [73]. This complicates the comparison of precessing systems because the orientation of the spin is now time dependent. This is not the only ambiguity present when comparing PN with NR; the definition of the spin is not necessarily the same [74].

We have attempted to account for any ambiguity in the definition of the spin orientation by allowing the frequency at which the spins are specified to vary; however, we acknowledge that this modification may smooth out model inaccuracies. This is similar to what was done in Ref. [22] where SEOBNRV3 was compared to a similar set of NR waveforms. In IMRPhenomPv2 and IMRPhenomPv3 we can fix the start frequency, $f_{\text {start }}$, and vary the spin-reference frequency $\left(f_{\text {ref }}\right)$; however, in the LALSuite implementation of SEOBNRV3 this is not possible and instead the spins are defined at the start frequency. Therefore we perform the optimization of the spin-reference frequency slightly differently between the phenom models and the EOB-NR model. For the phenom models we numerically optimize the overlap over $f_{\text {ref }}$ in the following range $[0.8,1.4] \times f_{\text {start }}$ and for SEOBNRv3 we numerically optimize the overlap over $f_{\text {start }}$ in the same range but if $1.4 f_{\text {start }}$ is greater than the maximum start frequency allowed by the EOB-NR generator, $f_{\max }^{\mathrm{EOB}}$, then we use this. ${ }^{6}$ For long waveforms the variation in the resulting match is less that $1 \%$; however, for shorter waveforms, such as SXS:BBH:0165, the variation can be as larger as $8 \%$.

Our NR signal waveforms only contain the $\ell=2$ multipoles, which is a good approximation for comparable mass systems $q \lesssim 3$ and for small inclinations where the effect of precession on the waveform is minimized. This assumption breaks down for some of the cases we consider here but we are primarily concerned with how faithful our new model is to a signal which contains the modes that we have modeled. We also want to keep separate systematic errors due to neglecting $\ell>2$ modes and those due to inaccuracies in modeling the $\ell=2$ modes. We compute the

\footnotetext{
${ }^{6} f_{\max }^{\mathrm{EOB}}$ is equal to the orbital frequency at a separation of $10 M$.
} 

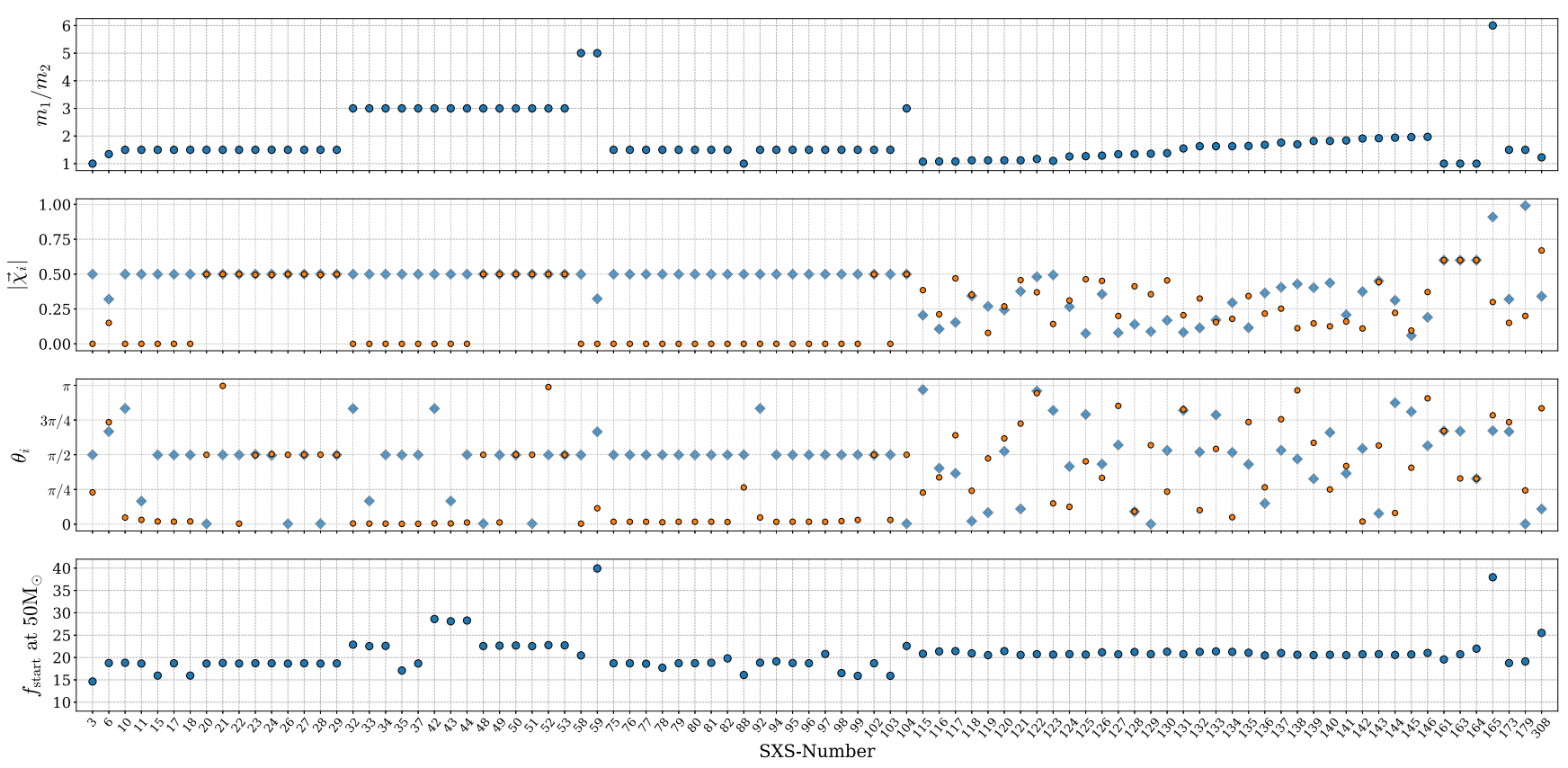

FIG. 3. The precessing parameter space we probe using the SXS public catalogue. The $\mathrm{x}$ axis is the SXS catalogue number. The four panels (from top to bottom) show the mass ratio $q=m_{1} / m_{2}$, the dimensionless spin magnitudes $\left|\vec{\chi}_{i}\right|$, the polar angle between the Newtonian orbital angular momentum and the individal BH spin vectors $\theta_{i}$ where $i=1,2$ for the larger and smaller BH respectively and finally the last panel plots the start GW frequency for each simulation when scaled to a total mass of $50 \mathrm{M}_{\odot}$, where the primary and secondary BHs are plotted as blue diamonds and orange circles respectively.

match assuming the theoretical design sensitivity of one of the LIGO detectors (zero detuned high power PSD [75]) and starting from a low frequency cutoff of $10 \mathrm{~Hz}$ until the end of the waveform. We compute the match over a range of total masses spanning from 20 to $200 M_{\odot}$. If the NR waveform is not long enough to start at $10 \mathrm{~Hz}$ then we use the starting frequency of the NR waveform as the low frequency cutoff in the match integral.

\section{B. NR catalogue}

We use the SXS public catalogue [76,77] of NR waveforms as our validation set consisting of 90 precessing waveforms with mass ratios between 1:1 and 1:6. Figure 3 presents an illustration of the parameter space covered. This set of NR waveforms mainly covers the mass-ratio space between $1: 1$ and 1:3 with only three waveforms above this: two at mass-ratio $1: 5$ and one at 1:6. This is clearly a heavily undersampled region of parameter space across NR groups (see other public catalogues from RIT $[78,79]$ and GaTech $[80,81])$. The vast majority of cases also have spin magnitudes that are $\leq 0.5$; however there is one case (SXS: BBH:0165) where $|\vec{\chi}|=0.9$. However, this is a short waveform of only six orbits in length. Again this highlights the need for longer NR simulations of BBHs with $|\vec{\chi}|>0.5$ across all mass ratios.

The bottom panel of Fig. 3 illustrates the length of the $\mathrm{NR}$ simulations. It shows the start frequency, in $\mathrm{Hz}$, of the $\ell=|m|=2 \mathrm{GW}$ multipole when the $\mathrm{BBH}$ system is scaled to $50 M_{\odot}$. In order for a NR waveform to be used in the analysis of LIGO-Virgo data, without hybridizing to $\mathrm{PN}$, the waveform needs to span the sensitive region of the detector; a typical start frequency for current instruments is between 20 and $25 \mathrm{~Hz}$ (see Fig. 2 of [6]). We see that for a BBH of total mass $50 M_{\odot}$, similar to GW150914 [3], many of these cases start at around $20 \mathrm{~Hz}$. Again there are a couple of outliers at mass ratios $1: 5$ and 1:6.

\section{Results}

Figure 4 shows the results of the orientation-averaged mismatch $(1-\overline{\mathcal{M}})$ calculation as a function of the total mass. Each row corresponds to a fixed template waveform labeled in the top left of each plot in the first column. From top to bottom each row shows results for IMRPhenomPv2, IMRPhenOmPV3 and SEOBNRv3. From left to right each column corresponds to the three different inclination angles we tested $0, \pi / 3$ and $\pi / 2$ respectively.

First we note that, for all three models, the majority of cases have mismatches smaller that 3\% and many smaller than $1 \%$. This is in agreement with the findings of Ref. [22]. Interestingly we find that the mismatch varies weakly with inclination angle indicating that the models perform well even when precession effects are amplified. One of the key results is the superior performance of IMRPhenomPv3 for total masses between 20 and $50 M_{\odot}$ where all but one case (SXS:BBH:0165 is discussed below) has mismatches $\lesssim 1 \%$. We attribute this to the more 


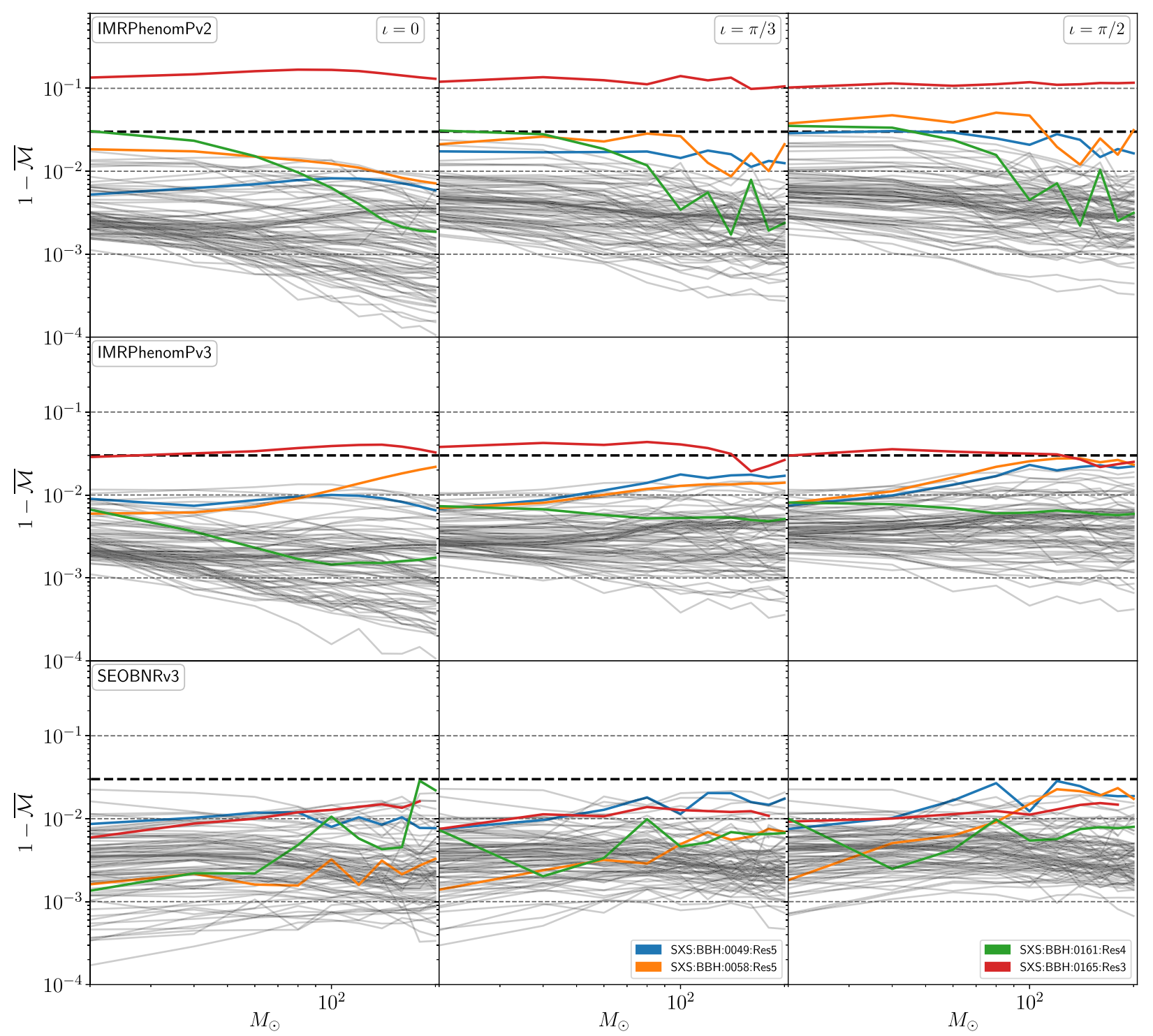

FIG. 4. The results of the comparison between IMRPhenomPv2 (first row), IMRPhenomPv3 (second row) and SEOBNRV3 (third row) and the precessing NR simulations from the public SXS catalogue. Each column shows the results for $\iota=(0, \pi / 3, \pi / 2)$, from left to right, where $\imath$ is the angle between the Newtonian orbital angular momentum $\vec{L}$ and the line of sight, at the start frequency. The figure shows the orientation-averaged mismatch $(1-\overline{\mathcal{M}})$ as a function of the total mass (log scale). Cases which have a maximum mismatch greater than $3 \%$ are colored and presented in Table I.

accurate and reliable new model of the precession dynamics of $[37,38]$.

To highlight regions of parameter space where current models are least accurate we have compiled Table I, which lists the SXS catalogue number, their mass ratio and initial spin components if at any point the mismatch is greater than $3 \%$. In the table we report the orientation-averaged mismatch averaged over all total masses for each inclination angle. We also color these cases in Fig. 4.

Our expectation that higher mass-ratio systems will yield the least accurate results, due to the fact that precession is based on a PN model and not calibrated to NR, is borne out in our results. Out of all the worst cases, with the exception of SXS:BBH:0161, the worst cases have mass ratios $\geq 3$. SXS:BBH:0161 is the only equal-mass outlier with worst mismatch marginally greater than 3\% for IMRPhenomPv2. For IMRPhenomPv3 and SEOBNRV3 the accuracy is better than $1 \%$ for the vast majority of cases.

The case with the highest mismatch, across all templates, is SXS:BBH:0165. This is a mass-ratio 1:6 system with initial spins $S_{1}=(0.74,0.19,-0.50)$ and $S_{2}=(-0.19,0$, -0.23 ) with a length of $\sim 6.5$ orbits. IMRPhenomPv2 has a best mismatch of $\sim 12 \%$. IMRPhenomPv 3 substantially improves upon this with a worst mismatch of $\sim 3.6 \%$. SEOBNRv3 performs well achieving a worst mismatch of $\sim 1.3 \%$. Given that the precession, in all three IMR models, 
TABLE I. Percentage mismatches averaged over all total masses for each the three inclination angles (rad) considered. All cases that have a mismatch larger than 3\% are shown. The first column shows the SXS ID number with the mass ratio and initial spin vectors in parentheses.

\begin{tabular}{|c|c|c|c|c|c|c|c|c|c|}
\hline \multirow{2}{*}{$\frac{\text { Waveform model }}{\text { SXS:BBH:ID }}$} & \multicolumn{3}{|c|}{ IMRPhenomPv2 } & \multicolumn{3}{|c|}{ IMRPhenomPv3 } & \multicolumn{3}{|c|}{ SEOBNRV3 } \\
\hline & 0 & $\pi / 3$ & $\pi / 2$ & 0 & $\pi / 3$ & $\pi / 2$ & 0 & $\pi / 3$ & $\pi / 2$ \\
\hline $0049\left[q=3, \vec{S}_{1}=(0.5,0,0), \vec{S}_{2}=(0,0,0.5)\right]$ & 0.7 & 1.5 & 2.4 & 0.9 & 1.4 & 1.8 & 1.0 & 1.5 & 1.8 \\
\hline $0058\left[q=5, \vec{S}_{1}=(0.5,0,0), \vec{S}_{2}=(0,0,0)\right]$ & 1.2 & 2.0 & 3.3 & 1.3 & 1.2 & 2.2 & 0.2 & 0.5 & 1.4 \\
\hline $0161\left[q=1, \vec{S}_{1}=(0.52,0,-0.3), \vec{S}_{2}=(0.52,0,-0.3)\right]$ & 1.0 & 1.1 & 1.4 & 0.2 & 0.6 & 0.7 & 0.8 & 0.6 & 0.7 \\
\hline $0165\left[q=6, \vec{S}_{1}=(0.74,0.19,-0.50), \vec{S}_{2}=(-0.19,0,-0.23)\right]$ & 15.4 & 12.5 & 12.1 & 3.6 & 3.5 & 3.0 & 1.2 & 1.1 & 1.3 \\
\hline
\end{tabular}

is modeled with uncalibrated PN or EOB calculations, it is not surprising that the region of parameter space where the models do worst comes from high mass ratios and high spin magnitudes. This is, by far, the case with the most dramatic improvement. In terms of its place in parameter space it has the largest mass-ratio and spin magnitude.

Out of the two mass-ratio 1:5 systems one of them has a worst mismatch larger than 3\%. This case, SXS:BBH:0058, is also the longest mass-ratio $1: 5$ case with approximately 28 orbits. We improve from a worst mismatch of $3.3 \%$ with IMRPhenomPv2 to $2.2 \%$ with IMRPhenomPv3. SEOBNRV3 shows good agreement with a worst average mismatch of $\sim 1.4 \%$.

For cases with mass ratio 1:3 we find that only 1 (SXS: BBH:0049) out of the 15 cases has a mismatch larger than $3 \%$. The mismatch is worst for IMRPhenomPv2 and only goes marginally above 3 for the $l=\pi / 2$ case where we expect the effects of precession to be most pronounced. With IMRPhenomPv 3 we improve, across all inclinations, with average mismatches between $1 \%$ and $2 \%$. We find a similar performance with SEOBNRV3.

Note however, that all the mass-ratio $1: 3$ and $1: 5$ systems have spin magnitudes $\leq 0.5$. Validating precessing waveform models at large spin magnitudes requires more NR simulations to be performed. We highlight again that these comparisons only include the $\ell=2$ multipoles in the signal waveform and for larger mass-ratio systems $(q \gtrsim 3)$; neglecting the higher multipoles is no longer a good approximation to the full GW signal.

\section{GW151226 ANALYSIS}

As a first application of the new IMRPhenomPv3 model we study whether the improved two-spin prescription allows for improved parameter extraction from existing GW signals. We focus on event GW151226 as it is the only system with strong support for at least one spinning $\mathrm{BH}$ $[4,54]$. Only recently, GW151226 has been analyzed with a precessing model that employs two-spin dynamics [82]. The source of GW151226 has a low-enough total mass that an analysis with SEOBNRV3 is computationally very challenging; simultaneously, GW151226 is so massive that the merger phase of the coalescence is in band, making an analysis with precessing inspiral-only models, such as SpinTaylorT4, incomplete.

The new IMRPhenomPV3 waveform model constructed here meets both criteria of computational efficiency and full coalescence description, allowing us to perform an efficient two-spin analysis of GW151226. We use the Bayesian inference code LALInferenceNest [83] and publicly available data from the LIGO Open Science Centre. We estimate the power spectral density noise using on-source data and the BayesWave algorithm $[84,85]$. We marginalize over detector calibration amplitude and phase uncertainty using values provided in [53]. We use a spin prior that is uniform in direction and magnitude up to 0.89 . In what follows, we present and compare results obtained using IMRPhenomPv2 using a reduced-order-quadrature approximation to the likelihood [39] and IMRPhenomPv3.

Table II presents our results for the intrinsic parameters. We quote the median value from the one-dimensional marginalized posterior and the associated 90\% symmetric credible interval. Noteworthy differences are a broader uncertainty in the primary mass resulting in IMRPhenomPv3 favoring a slightly more asymmetric system, although both models are within the statistical uncertainty of each other. Overall we find consistent results between the two models as well as the published LIGOVirgo Collaboration analysis [53]. We also have an estimate of the Bayes factor for a coherent signal across the two

TABLE II. Parameter table for GW151226. Masses are defined in the source frame. We quote the median and the $90 \%$ symmetric credible interval of the one-dimensional marginalized posterior distributions.

\begin{tabular}{lcc}
\hline \hline Parameter & IMRPhenomPv2 & IMRPhenomPv3 \\
\hline Primary mass: $m_{1}\left(M_{\odot}\right)$ & $13.68_{-3.13}^{+7.93}$ & $14.45_{-3.96}^{+10.23}$ \\
Secondary mass: $m_{2}\left(M_{\odot}\right)$ & $7.73_{-2.10}^{+2.41}$ & $7.35_{-2.52}^{+2.57}$ \\
Total mass: $M_{\text {tot }}\left(M_{\odot}\right)$ & $21.50_{-1.44}^{+5.48}$ & $21.85_{-1.77}^{+7.64}$ \\
Mass ratio: $q$ & $0.57_{-0.32}^{+0.36}$ & $0.51_{-0.31}^{+0.44}$ \\
Effective spin: $\chi_{\text {eff }}$ & $0.19_{-0.07}^{+0.18}$ & $0.20_{-0.09}^{+0.23}$ \\
Precession parameter: $\chi_{\mathrm{p}}$ & $0.44_{-0.28}^{+0.34}$ & $0.44_{-0.28}^{+0.35}$ \\
Log Bayes factor: $\log (\mathcal{B})$ & 50.707 & 51.291 \\
\hline \hline
\end{tabular}



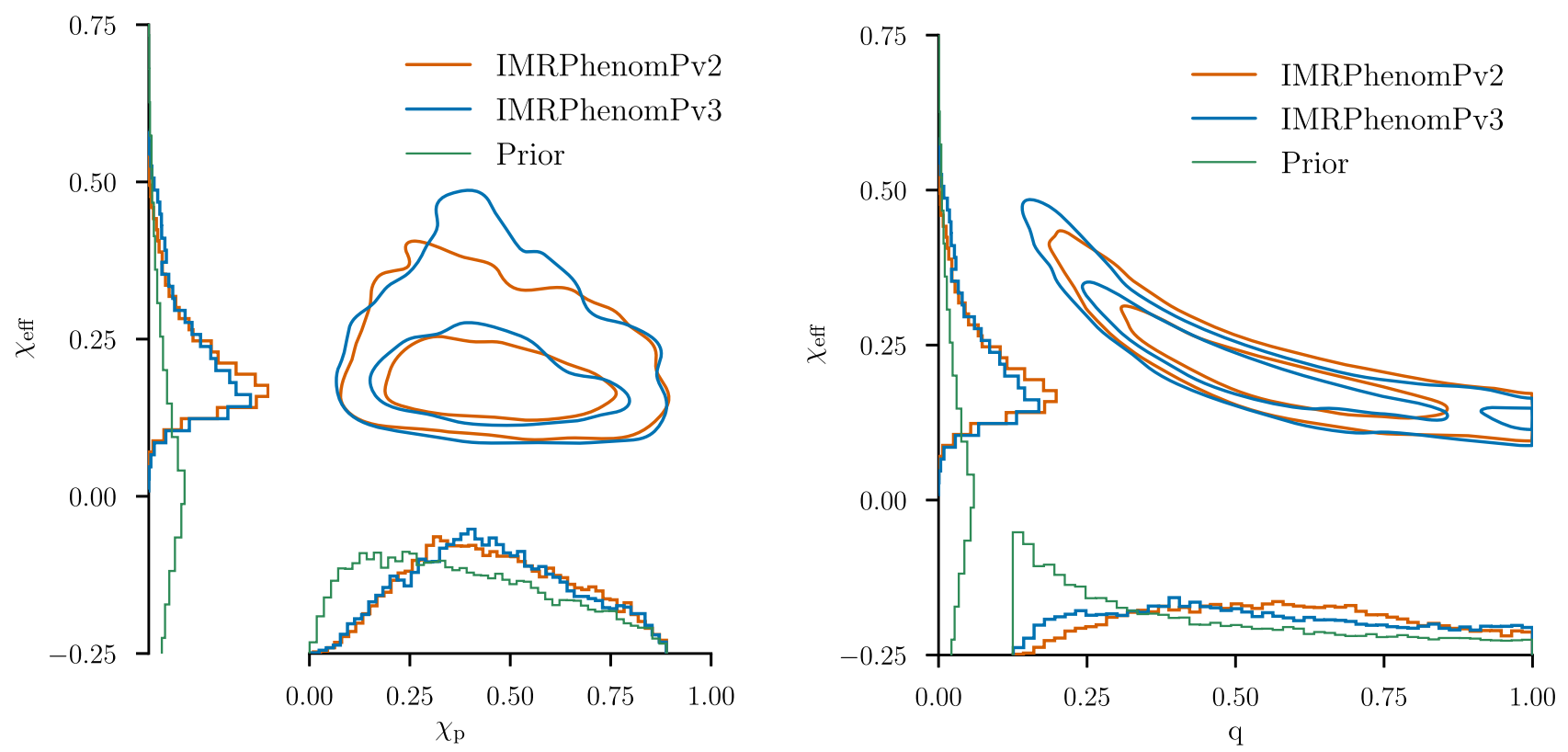

FIG. 5. Posteriors probability densities for $\chi_{\text {eff }}-\chi_{p}$ (left) and $\chi_{\text {eff }}-q$ (right) for GW151226 using IMRPhenomPv2 and IMRPhenomPv3. Despite the more accurate description of two-spin dynamics of IMRPhenomPv3 the posteriors are consistent, demonstrating the difficulty of measuring two-spin dynamics.

LIGO detectors versus an incoherent signal or noise. We find a slightly larger Bayes factor for IMRPhenomPv3 despite the extra degrees of freedom from the full two-spin description.

Figure 5 shows the two-dimensional posterior densities for $\chi_{\text {eff }}-\chi_{p}{ }^{7}$ (left) and $\chi_{\text {eff }}-q$ (right) obtained from both waveform models as well as the one-dimensional prior distributions. Both plots demonstrate broad agreement with IMRPhenomPv3 marginally favoring more unequal masses. This result is also consistent based on the comparisons to NR waveforms presented in the previous section. The posterior for $\chi_{p}$ is consistent with its prior, confirming the absence of evidence of spin precession in GW151226. Overall we find that a reanalysis of the merger event GW151226 with our new waveform model does not provide new insights into the nature of the source but instead reinforces our current understanding of the source.

The consistency of the results implies that two-spin precession effects do not impact this signal in agreement with previous studies predicting that aligned-two-spin effects are not easily measurable [86]. However, it is possible that certain binaries and orientations might make it possible to measure both spins simultaneously. This could be achieved, for example, with precessing models that also include higher multipoles modes, or with louder signals observed by more detectors.

\footnotetext{
${ }^{7}$ Note that IMRPhenomPv3 no longer explicitly uses the effective precession parameter $\chi_{p}$. However we show it here for direct comparison to IMRPhenomPv2 results.
}

\section{DISCUSSION}

We have presented an upgrade to the phenomenological model IMRPhenomPv2 called IMRPhenomPv3. This model predicts the GW polarizations computed using the dominant $\ell=|m|=2$ multipole in the coprecessing frame, from noneccentric merging $\mathrm{BBHs}$ with generically orientated spins. Our upgrade consists of replacing the model for the precession dynamics of [23] that was derived under the assumption of a single spin precessing $\mathrm{BBH}$ system with the more accurate analytic model from $[37,38]$ that contains two-spin effects.

We have validated our new model against a large set of precessing NR waveforms. Although our selection of NR waveforms is biased towards mass ratios $<3$ and spin magnitudes $<0.5$ we find that all three models considered generally perform well; however, we have identified a clear region in parameter space, mass ratios $>3$, where all models begin to loose accuracy. Encouragingly we find that IMRPhenomPV3 greatly outperforms the previous model for the most extreme case we considered, with the largest improvement being $\sim 12 \%$. This improvement suggests that IMRPhenomPv3 can be utilized in a much wider parameter space than IMRPhenomPv2 was found to be reliable. We emphasize the importance of NR to continue to push the capabilities of precessing BBH simulations that will allow more stringent tests of waveforms models and ultimately lead to more accurate waveform models being developed.

As a first application we reanalyzed GW151226 with our two-spin model and find consistent results compared with 
the single-spin model, thereby reinforcing our current inference on the nature of GW151226.

As our waveform model is analytic one can evaluate the model using a nonuniform grid of frequencies. This is essential for methods such as reduced order quadrature [39] and the multibanding technique of [87]. These methods can increase the computational efficiency of sampling-based parameter estimation by factors of 300 for low-mass systems.

We note that there are a number of physical effects that are ignored. We do not model any asymmetry between the positive and negative $m$ modes, which are responsible for large out-of-plane recoil kick velocities. In the underlying, aligned-spin model we do not include effects from higher order multipoles; however, we have recently developed a method to do this [32] and we are currently determining the accuracy of a precessing model that includes higher order multipoles in the coprecessing frame. Finally, the solutions to the spin-precession dynamics presented in Refs. [37,38] and, by extension, the new model we construct here assume that the direction of the total angular momentum is approximately fixed. Therefore they do not capture the effect of transitional precession [18].

\section{ACKNOWLEDGMENTS}

We thank Carl-Johan Haster for kindly supplying the PSD computed using BayesWave for the parameter estimation study of GW151226. We thank Sylvain Marsat for useful discussions. S. K. and F. O. acknowledge support by the Max Planck Society's Independent Research Group Grant. We thank the Atlas cluster computing team at AEI Hannover where this analysis was carried out. M. H. was supported by Science and Technology Facilities Council (STFC) Grant No. ST/L000962/1 and European Research Council Consolidator Grant No. 647839. This work made use of numerous open source computational packages such as python [88], NumPy, SciPy [89], Matplotlib [90] and the GW data analysis software library pycbc [91].
[1] J. Aasi et al. (LIGO Scientific Collaboration), Classical Quantum Gravity 32, 074001 (2015).

[2] F. Acernese et al. (VIRGO Collaboration), Classical Quantum Gravity 32, 024001 (2015).

[3] B. P. Abbott et al. (LIGO and Virgo Scientific Collaborations), Phys. Rev. Lett. 116, 061102 (2016).

[4] B. P. Abbott et al. (LIGO and Virgo Scientific Collaborations), Phys. Rev. Lett. 116, 241103 (2016).

[5] B. P. Abbott et al. (LIGO and Virgo Scientific Collaborations), Phys. Rev. Lett. 118, 221101 (2017).

[6] B. P. Abbott et al. (Virgo and LIGO Scientific Collaborations), Phys. Rev. Lett. 119, 141101 (2017).

[7] B. P. Abbott et al. (Virgo and LIGO Scientific Collaborations), Astrophys. J. 851, L35 (2017).

[8] B. P. Abbott et al. (Virgo and LIGO Scientific Collaborations), Phys. Rev. Lett. 119, 161101 (2017).

[9] B. P. Abbott et al. (GROND, SALT Group, OzGrav, DFN, INTEGRAL, Virgo, Insight-Hxmt, MAXI Team, FermiLAT, J-GEM, RATIR, IceCube, CAASTRO, LWA, ePESSTO, GRAWITA, RIMAS, SKA South Africa/MeerKAT, H.E.S.S., 1M2H Team, IKI-GW Follow-up, Fermi GBM, Pi of Sky, DWF (Deeper Wider Faster Program), Dark Energy Survey, MASTER, AstroSat Cadmium Zinc Telluride Imager Team, Swift, Pierre Auger, ASKAP, VINROUGE, JAGWAR, Chandra Team at McGill University, TTUNRAO, GROWTH, AGILE Team, MWA, ATCA, AST3, TOROS, Pan-STARRS, NuSTAR, ATLAS Telescopes, BOOTES, CaltechNRAO, LIGO Scientific, High Time Resolution Universe Survey, Nordic Optical Telescope, Las Cumbres Observatory Group, TZAC Consortium, LOFAR, IPN, DLT40, Texas Tech University, HAWC, ANTARES, KU, Dark Energy Camera GW-EM, CALET, Euro VLBI Team, ALMA), Astrophys. J. 848, L12 (2017).
[10] C. Messick et al., Phys. Rev. D 95, 042001 (2017).

[11] T. Dal Canton et al., Phys. Rev. D 90, 082004 (2014).

[12] S. A. Usman et al., Classical Quantum Gravity 33, 215004 (2016).

[13] A. H. Nitz, T. Dent, T. Dal Canton, S. Fairhurst, and D. A. Brown, Astrophys. J. 849, 118 (2017).

[14] S. Klimenko, G. Vedovato, M. Drago, F. Salemi, V. Tiwari, G. A. Prodi, C. Lazzaro, K. Ackley, S. Tiwari, C. F. Da Silva, and G. Mitselmakher, Phys. Rev. D 93, 042004 (2016).

[15] C. Cutler and E. E. Flanagan, Phys. Rev. D 49, 2658 (1994).

[16] J. Veitch et al., Phys. Rev. D 91, 042003 (2015).

[17] B. P. Abbott et al. (Virgo and LIGO Scientific Collaborations), Phys. Rev. Lett. 116, 241102 (2016).

[18] T. A. Apostolatos, C. Cutler, G. J. Sussman, and K. S. Thorne, Phys. Rev. D 49, 6274 (1994).

[19] L. E. Kidder, Phys. Rev. D 52, 821 (1995).

[20] Y. Pan, A. Buonanno, A. Taracchini, L. E. Kidder, A. H. Mroué, H. P. Pfeiffer, M. A. Scheel, and B. Szilágyi, Phys. Rev. D 89, 084006 (2014).

[21] A. Taracchini et al., Phys. Rev. D 89, 061502 (2014).

[22] S. Babak, A. Taracchini, and A. Buonanno, Phys. Rev. D 95, 024010 (2017).

[23] M. Hannam, P. Schmidt, A. Bohé, L. Haegel, S. Husa, F. Ohme, G. Pratten, and M. Pürrer, Phys. Rev. Lett. 113, 151101 (2014).

[24] L. Santamaría, F. Ohme, P. Ajith, B. Brügmann, N. Dorband, M. Hannam, S. Husa, P. Mösta, D. Pollney, C. Reisswig, E. L. Robinson, J. Seiler, and B. Krishnan, Phys. Rev. D 82, 064016 (2010).

[25] S. Husa, S. Khan, M. Hannam, M. Pürrer, F. Ohme, X. J. Forteza, and A. Bohé, Phys. Rev. D 93, 044006 (2016). 
[26] S. Khan, S. Husa, M. Hannam, F. Ohme, M. Pürrer, X. Jiménez Forteza, and A. Bohé, Phys. Rev. D 93, 044007 (2016).

[27] T. D. Abbott et al. (Virgo and LIGO Scientific Collaborations), Phys. Rev. X 6, 041014 (2016).

[28] B. P. Abbott et al. (Virgo and LIGO Scientific Collaborations), Classical Quantum Gravity 34, 104002 (2017).

[29] R. Sturani, S. Fischetti, L. Cadonati, G. M. Guidi, J. Healy, D. Shoemaker, and A. Viceré, J. Phys. Conf. Ser. 243, 012007 (2010).

[30] Y. Pan, A. Buonanno, M. Boyle, L. T. Buchman, L. E. Kidder, H. P. Pfeiffer, and M. A. Scheel, Phys. Rev. D 84, 124052 (2011).

[31] A. K. Mehta, C. K. Mishra, V. Varma, and P. Ajith, Phys. Rev. D 96, 124010 (2017).

[32] L. London, S. Khan, E. Fauchon-Jones, C. García, M. Hannam, S. Husa, X. Jiménez-Forteza, C. Kalaghatgi, F. Ohme, and F. Pannarale, Phys. Rev. Lett. 120, 161102 (2018).

[33] R. Cotesta, A. Buonanno, A. Bohé, A. Taracchini, I. Hinder, and S. Ossokine, Phys. Rev. D 98, 084028 (2018).

[34] S. E. Field, C. R. Galley, J. S. Hesthaven, J. Kaye, and M. Tiglio, Phys. Rev. X 4, 031006 (2014).

[35] J. Blackman, S. E. Field, M. A. Scheel, C. R. Galley, C. D. Ott, M. Boyle, L. E. Kidder, H. P. Pfeiffer, and B. Szilágyi, Phys. Rev. D 96, 024058 (2017).

[36] Y. E. Setyawati, F. Ohme, and S. Khan, Phys. Rev. D 99, 024010 (2019).

[37] K. Chatziioannou, A. Klein, N. Yunes, and N. Cornish, Phys. Rev. D 95, 104004 (2017).

[38] K. Chatziioannou, A. Klein, N. Cornish, and N. Yunes, Phys. Rev. Lett. 118, 051101 (2017).

[39] R. Smith, S. E. Field, K. Blackburn, C.-J. Haster, M. Pürrer, V. Raymond, and P. Schmidt, Phys. Rev. D 94, 044031 (2016).

[40] P. Schmidt, M. Hannam, S. Husa, and P. Ajith, Phys. Rev. D 84, 024046 (2011).

[41] R. O'Shaughnessy, B. Vaishnav, J. Healy, Z. Meeks, and D. Shoemaker, Phys. Rev. D 84, 124002 (2011).

[42] L. Pekowsky, R. O'Shaughnessy, J. Healy, and D. Shoemaker, Phys. Rev. D 88, 024040 (2013).

[43] A. Buonanno, Y.-b. Chen, and M. Vallisneri, Phys. Rev. D 67, 104025 (2003); 74, 029904(E) (2006).

[44] P. Schmidt, M. Hannam, and S. Husa, Phys. Rev. D 86, 104063 (2012).

[45] M. Boyle, R. Owen, and H. P. Pfeiffer, Phys. Rev. D 84, 124011 (2011).

[46] P. Schmidt, F. Ohme, and M. Hannam, Phys. Rev. D 91, 024043 (2015).

[47] S. Droz, D. J. Knapp, E. Poisson, and B. J. Owen, Phys. Rev. D 59, 124016 (1999).

[48] https://dcc.ligo.org/LIGO-T1500602.

[49] X. Jiménez-Forteza, D. Keitel, S. Husa, M. Hannam, S. Khan, and M. Pürrer, Phys. Rev. D 95, 064024 (2017).

[50] B. P. Abbott et al. (LIGO Scientific and Virgo Collaborations), Phys. Rev. Lett. 116, 131103 (2016).

[51] B. P. Abbott et al. (LIGO Scientific and Virgo Collaborations), Phys. Rev. Lett. 116, 241102 (2016).

[52] B. P. Abbott et al. (LIGO Scientific and Virgo Collaborations), Phys. Rev. Lett. 116, 221101 (2016).
[53] B. P. Abbott et al. (LIGO Scientific and Virgo Collaborations), Phys. Rev. X 6, 041015 (2016).

[54] S. Vitale, D. Gerosa, C.-J. Haster, K. Chatziioannou, and A. Zimmerman, Phys. Rev. Lett. 119, 251103 (2017).

[55] T. Dietrich et al., Phys. Rev. D 99, 024029 (2019).

[56] B. P. Abbott et al. (Virgo and LIGO Scientific Collaborations), Phys. Rev. X 9, 011001 (2019).

[57] B. P. Abbott et al. (Virgo and LIGO Scientific Collaborations), Phys. Rev. Lett. 121, 161101 (2018).

[58] P. Schmidt, Studying and modelling the complete gravitational-wave signal from precessing black hole binaries, https://orca.cf.ac.uk/64062/.

[59] M. Kesden, D. Gerosa, R. O’Shaughnessy, E. Berti, and U. Sperhake, Phys. Rev. Lett. 114, 081103 (2015).

[60] A. Klein, N. Cornish, and N. Yunes, Phys. Rev. D 88, 124015 (2013).

[61] K. Chatziioannou, A. Klein, N. Yunes, and N. Cornish, Phys. Rev. D 88, 063011 (2013).

[62] C. M. Bender and S. A. Orszag, Advanced Mathematical Methods for Scientists and Engineers 1, Asymptotic Methods and Perturbation Theory (Springer, New York, 1999).

[63] A. Klein, N. Cornish, and N. Yunes, Phys. Rev. D 90, 124029 (2014).

[64] M. Cabero, A. B. Nielsen, A. P. Lundgren, and C. D. Capano, Phys. Rev. D 95, 064016 (2017).

[65] F. Hofmann, E. Barausse, and L. Rezzolla, Astrophys. J. 825, L19 (2016).

[66] X. Jiménez-Forteza, D. Keitel, S. Husa, M. Hannam, S. Khan, and M. Pürrer, Phys. Rev. D 95, 064024 (2017).

[67] V. Varma, D. Gerosa, L. C. Stein, F. Hébert, and H. Zhang, Phys. Rev. Lett. 122, 011101 (2019).

[68] B. Bruegmann, J. A. Gonzalez, M. Hannam, S. Husa, and U. Sperhake, Phys. Rev. D 77, 124047 (2008).

[69] I. Harry, S. Privitera, A. Bohé, and A. Buonanno, Phys. Rev. D 94, 024012 (2016).

[70] I. Harry, J. Calderón Bustillo, and A. Nitz, Phys. Rev. D 97, 023004 (2018).

[71] https://wiki.ligo.org/DASWG/LALSuite.

[72] P. Schmidt, I. W. Harry, and H.P. Pfeiffer, arXiv: 1703.01076.

[73] E. Hamilton and M. Hannam, Phys. Rev. D 98, 084018 (2018).

[74] R. Owen, A. S. Fox, J. A. Freiberg, and T. P. Jacques, Phys. Rev. D 99, 084031 (2019).

[75] https://dcc.ligo.org/LIGO-T0900288/public.

[76] A. H. Mroué, M. A. Scheel, B. Szilágyi, H. P. Pfeiffer, M. Boyle, D. A. Hemberger, L. E. Kidder, G. Lovelace, S. Ossokine, N. W. Taylor, A. Zenginoğlu, L. T. Buchman, T. Chu, E. Foley, M. Giesler, R. Owen, and S. A. Teukolsky, Phys. Rev. Lett. 111, 241104 (2013).

[77] https://data.black-holes.org/waveforms/index.html.

[78] J. Healy, C. O. Lousto, Y. Zlochower, and M. Campanelli, Classical Quantum Gravity 34, 224001 (2017).

[79] http://ccrg.rit.edu/ RITCatalog/.

[80] K. Jani, J. Healy, J. A. Clark, L. London, P. Laguna, and D. Shoemaker, Classical Quantum Gravity 33, 204001 (2016).

[81] http://www.einstein.gatech.edu/catalog/.

[82] B. P. Abbott et al. (LIGO Scientific and Virgo Collaborations), arXiv:1811.12907. 
[83] J. Veitch, V. Raymond, B. Farr, W. Farr, P. Graff, S. Vitale et al., Phys. Rev. D 91, 042003 (2015).

[84] T. B. Littenberg and N. J. Cornish, Phys. Rev. D 91, 084034 (2015).

[85] N. J. Cornish and T. B. Littenberg, Classical Quantum Gravity 32, 135012 (2015).

[86] M. Pürrer, M. Hannam, and F. Ohme, Phys. Rev. D 93, 084042 (2016).
[87] S. Vinciguerra, J. Veitch, and I. Mandel, Classical Quantum Gravity 34, 115006 (2017).

[88] https://www.python.org/.

[89] E. Jones, T. Oliphant, P. Peterson et al., SciPy: Open source scientific tools for Python, 2001, http://www.scipy.org/.

[90] J. D. Hunter, Comput. Sci. Eng. 9, 90 (2007).

[91] A. Nitz et al., pycbc software, 2018, http://dx.doi.org/10 .5281/zenodo.3265452. 